\title{
State of the art of haptic feedback in virtual reality in manufacturing
}

\begin{abstract}
This paper discusses the application of haptic feedback in Virtual Reality to enhance user performance in manufacturing. Haptic interfaces have the potential to enhance communication and interaction via the computer enabling affective expressive interpersonal communication and enriching interaction by haptic feedback. The aim of this study was to investigate and develop a better understanding of using haptic feedback in manufacturing.
\end{abstract}

Keyword: Haptic feedback; Virtual reality; Manufacturing 